\title{
Workflow and Scan Protocol Considerations for Integrated Whole-Body PET/MRI in Oncology
}

\author{
Axel Martinez-Möller*1, Matthias Eiber*2 ${ }^{*}$ Stephan G. Nekolla ${ }^{1}$, Michael Souvatzoglou ${ }^{1}$, Alexander Drzezga ${ }^{1}$, \\ Sibylle Ziegler ${ }^{1}$, Ernst J. Rummeny ${ }^{2}$, Markus Schwaiger ${ }^{1}$, and Ambros J. Beer ${ }^{1}$ \\ ${ }^{I}$ Department of Nuclear Medicine, Technische Universität München, Munich, Germany; and ${ }^{2}$ Department of Radiology, Technische \\ Universität München, Munich, Germany
}

Learning Objectives: On successful completion of this activity, participants should be able to describe (1) special requirements for planning whole-body PET/MRI examinations; (2) general aspects of whole-body PET/MRI protocols in oncology; and (3) possible artifacts in PET/MRI.

Financial Disclosure: An author of this article is a meeting participant or lecturer for Siemens AG. No other relevant relationships that could be perceived as a real or apparent conflict of interest were reported.

CME Credit: SNMMI is accredited by the Accreditation Council for Continuing Medical Education (ACCME) to sponsor continuing education for physicians. SNMMI designates each JNM continuing education article for a maximum of 2.0 AMA PRA Category 1 Credit. Physicians should claim only credit commensurate with the extent of their participation in the activity. For CE credit, participants can access this activity through the SNMMI Web site (http:// www.snmmi.org/ce_online) through September 2013.

Integrated PET/MRI systems open exciting possibilities for clinical and research applications. However, compared with $\mathrm{PET} / \mathrm{CT}, \mathrm{PET} / \mathrm{MRI}$ is a complex technique resulting in new problems and challenges, especially regarding workflow, scan protocols, and data analysis. This complexity applies in particular to examinations in oncology with partial- or whole-body coverage extending over several bed positions. Unlike diagnostic PET/CT, for which the clinical CT protocols can largely be copied from stand-alone CT, the design of a diagnostic MRI protocol for partial- or whole-body coverage is more complex and has to be adapted to the special requirements of PET/MRI to be both time-efficient and comprehensive. Here, we describe basic considerations concerning workflow, imaging protocols, and image analysis for whole-body PET/MRI in oncology, based on our experience with the first integrated PET/MRI scanner. The aim is to fully and optimally make use of the combined PET/MRI measurements in oncology, including identifying and reducing image artifacts as well as optimizing workflow beyond the mere fusion of 2 image datasets.

Key Words: PET/MR; oncology; workflow; hybrid imaging

J Nucl Med 2012; 53:1415-1426

DOI: 10.2967/jnumed.112.109348

$\mathbf{T}$ he success of combined PET and CT has demonstrated the clinical value of multimodality imaging technology, providing both anatomic and molecular information within a single imaging session. However, whereas CT and espe-

\footnotetext{
Received May 28, 2012; revision accepted Jul. 20, 2012.

For correspondence or reprints contact: Ambros J. Beer, Department of Nuclear Medicine, Technische Universität München, Ismaningerstrasse 22, 81675 Munich, Germany.

E-mail: ambros.beer@tum.de

Published online Aug. 9, 2012.

COPYRIGHT (C 2012 by the Society of Nuclear Medicine and Molecular Imaging, Inc.

${ }^{*}$ Contributed equally to this work.
}

cially modern multislice CT are a powerful imaging tool, they still have shortcomings, such as low soft-tissue contrast when compared with MRI. Thus, the combination of PET and MRI might be clinically advantageous over PET/ CT for some indications. Moreover, because MRI has more potential for functional and molecular imaging than does CT, the combination of PET with MRI offers completely new opportunities for research applications and for molecular imaging in general.

As a consequence, a lot of effort has been put into the development of combined PET and MRI. The first preclinical combined PET/MRI systems were introduced in the 1990s (1-3). Clinically, a PET insert for a standard 3.0-T MRI scanner for simultaneous PET/MRI of the brain (BrainPET; Siemens) was the first successful approach showing the potential of truly simultaneous PET/MRI in the clinical arena (4). Finally, a decade after the commercial introduction of PET/CT, whole-body PET/MRI scanners have now been introduced on the market for clinical use.

There are currently solutions for combined PET and MRI from 3 vendors. GE Healthcare proposes having PET/CT and MRI scanners placed in neighboring rooms, with the ability to shuttle a patient bed between the rooms; each scanner can be used separately as a stand-alone system as well, or they can be used consecutively on the same patient, resulting in a trimodality approach. Philips Healthcare offers a sequential PET/MRI scanner named Ingenuity TF PET/MRI, with PET and MRI scanners being placed beside each other in a single room, and a patient bed capable of rotating $180^{\circ}$ being used to transfer the patient from one scanner into the other (5). Siemens Healthcare produces an integrated PET/MRI scanner named Biograph mMR, with the PET detector ring placed within the MRI main magnet using MRI-compatible PET photodetectors (6). 
The challenge now is how to exploit the potential of this new modality, which currently represents an expensive diagnostic imaging device. As of today, it is entirely unknown whether PET/MRI will provide incremental diagnostic accuracy, an incremental impact on management, or an incremental impact on patient outcome compared with PET/CT. However, before these questions can be adequately addressed, scan protocols have to be designed that are both time-efficient and comprehensive, as will be discussed in this report. The experience described is based on our work with an integrated system from a single vendor (Biograph mMR). However, the topics and issues described here are expected to be largely applicable to upcoming integrated systems from other vendors as well.

Integrated PET/MRI has raised several major questions, among them the time and cost efficiency of the systemcurrently an unsolved issue $(7,8)$. In neurologic and, to a lesser extent, cardiologic applications, which might strongly profit from hybrid PET/MRI, this question is usually less complex, as only a single bed position is examined $(9,10)$. The scanning time is then determined by the duration of either the PET or the MRI examination, and there is no need for a second scan in a separate scanner. The time efficiency of integrated PET/MRI is thus evident in this scenario. However, it would be incorrect to assume the same for an oncologic PET/MRI examination, in which an extended body area is usually investigated. Although each bed position in oncologic PET usually requires the same time (e.g., $3 \mathrm{~min}$ ), this is not necessarily true for MRI, for which different parts of the body might require different MRI sequences and thus a different amount of time to be examined adequately. For advanced molecular imaging applications or MRI spectroscopy, the time needed for the MRI part might be even longer than for routine protocols and might easily exceed $60 \mathrm{~min}$.

This work describes basic considerations for whole-body oncologic PET/MRI using an integrated system in clinical routine, with the aim of helping future users optimize their workflow and imaging protocols. This is a work in progress, and with growing experience the considerations and protocols presented here will need to be continuously adapted and improved.

\section{WORKFLOW AND LOGISTIC CONSIDERATIONS}

This section presents some basic but nevertheless important considerations concerning the workflow and logistics that need to be considered before an actual PET/ MRI scan is performed. Although such details as patient scheduling, preparation, and positioning may seem like common knowledge, the MRI-related aspects may be of interest to the nuclear medicine community, as may the PET aspects to the radiology community. Physicians and technologists from both communities will encounter new challenges with PET/MRI, compared with standalone MRI, PET, and PET/CT (Table 1).

\section{Patient Preparation Before Examination}

The patient preparation for PET/MRI will be basically the same as that for a PET or PET/CT examination. In the case of ${ }^{18} \mathrm{~F}-\mathrm{FDG}$, this includes prior fasting, control of the glucose level, frequent hydration, and having the patient rest between ${ }^{18} \mathrm{~F}-\mathrm{FDG}$ administration and imaging to minimize muscle uptake $(11,12)$. For claustrophobic patients, slight sedation such as with low doses of oral benzodiazepines is in our experience needed substantially more often for oncologic PET/MRI than for PET/CT, because of the smaller diameter and longer tunnel of the MRI scanner (13). Potential contraindications to MRI (e.g., non-MRI-compatible pacemakers or artificial heart valves) have to be checked in addition to the usual contraindications to PET, such as pregnancy.

\section{In-Bed Patient Preparation}

The patient should be positioned comfortably to minimize motion, with any necessary positioning aids, just as in standalone MRI or PET. What is new compared with standalone MRI is that care has to be taken that any additional positioning aids do not result in significant attenuation of the 511-keV photons (14). In PET/MRI, surface coils are used to increase MRI image quality and reduce imaging time, just as in standalone MRI. However, because the surface coils may cause additional attenuation of the 511$\mathrm{keV}$ photons, only dedicated coils approved for PET/MRI should be used. Patient preparation occurs after ${ }^{18} \mathrm{~F}-\mathrm{FDG}$ administration and takes substantially longer than for PET/CT and therefore has to be done as quickly as possible by trained personnel to minimize the radiation burden to the staff.

\section{OPTIMIZATION OF PET/MRI PROTOCOLS FOR ONCOLOGIC INDICATIONS}

\section{General Aspects}

Whole-body PET/MRI protocols all share some common aspects. First, MRI localizers have to be acquired (corresponding to the scout scan or topogram in PET/CT) to plan the subsequent acquisition and, in particular, to define the axial range for the joint PET/MRI examination. PET works in the so-called step-and-shoot mode, in which the 2 main acquisition parameters to be defined are the number of bed positions and the acquisition time for each. In the scanner we used, the axial range of a single bed position is $25.8 \mathrm{~cm}$, with a $6.1-\mathrm{cm}$ overlap between adjacent bed positions. The acquisition time can be kept similar to that in PET/CT, typically $2-4$ min per bed position; however, this time can also be increased to make use of the potentially longer interval required for the simultaneous MRI acquisitions. In principle, it is also feasible to acquire PET and MRI data while the patient bed is continuously moving, as recently described for combined PET/MRI (15). This extremely interesting approach might substantially facilitate the workflow for whole-body PET/ MRI but is not yet routinely integrated in currently used PET/MRI systems; thus, we will not cover this subject in more detail in this article. 
TABLE 1

Technical Aspects and Differences between PET and MRI Relevant for Optimizing Protocols in Integrated PET/MRI

\begin{tabular}{|c|c|c|}
\hline & PET & MRI \\
\hline \multirow[t]{3}{*}{ Patient schedule } & Rigorous patient schedule & Flexible patient schedule \\
\hline & Acquisition at fixed time after injection & $\begin{array}{l}\text { Additional imaging possible, depending on } \\
\text { findings or motion }\end{array}$ \\
\hline & High predictability of acquisition time & \\
\hline \multirow[t]{3}{*}{ Prescan preparation } & Tracer administration & $\begin{array}{l}\text { Checking of potential contraindications and } \\
\text { renal insufficiency when gadolinium is used }\end{array}$ \\
\hline & Resting of patient during uptake time & Removal of all metal \\
\hline & $\begin{array}{l}\text { With }{ }^{18} \mathrm{~F}-\mathrm{FDG} \text {, previous fasting and } \\
\text { glucose level measurement }\end{array}$ & \\
\hline \multirow[t]{2}{*}{ In-bed patient preparation } & Very fast; only uploading of patient onto table & Placement of surface coils and headphones \\
\hline & Radiation exposure for staff while near patients & Time-consuming (3-5 min) \\
\hline \multirow[t]{2}{*}{ Field of view } & $45-60 \mathrm{~cm}$ transaxially & $35-45 \mathrm{~cm}$ transaxially \\
\hline & $15-21 \mathrm{~cm}$ axially & Up to $50 \mathrm{~cm}$ axially \\
\hline \multirow{4}{*}{ Planning } & Straightforward & Experience for fine-tuning \\
\hline & Defining of field of view and time per bed position & Planning throughout scan \\
\hline & $\begin{array}{l}\text { Planning by operator at start, with no further } \\
\text { interaction }\end{array}$ & Frequent interaction with radiologist \\
\hline & & Often oblique plane acquisitions \\
\hline \multirow[t]{2}{*}{ Acquisition } & Step and shoot & Isocenter for optimal image quality \\
\hline & Overlapping of beds & $\begin{array}{l}\text { Occasional need for bed motion even in } \\
\text { examination of single organs }\end{array}$ \\
\hline \multirow{2}{*}{$\begin{array}{l}\text { Respiratory } \\
\text { motion }\end{array}$} & Production of blurring & Frequent production of artifacts \\
\hline & $\begin{array}{l}\text { Possibility of respiratory gating; increase of } \\
\text { acquisition time to preserve image quality }\end{array}$ & $\begin{array}{l}\text { Possibility of breath-hold acquisition } \\
\text { or respiratory gating }\end{array}$ \\
\hline
\end{tabular}

Attenuation data in PET/MRI are derived from the MRI scan $(16,17)$. For each bed position, the MRI sequence for attenuation correction (AC) purposes is acquired first. The sequence used in the integrated PET/MRI system is a 2-point Dixon volume-interpolated breath-hold examination (VIBE). A separate breath-hold acquisition takes place at each bed position, and imaging takes place at a rate of $19 \mathrm{~s}$ per bed position. This sequence is preceded by scanning preparations that include shimming to optimize the homogeneity of the magnetic field ( $\sim 40 \mathrm{~s})$. The MRI data are then segmented to identify air, lung tissue, fatty tissue, and watery tissue as required for AC (18). The 2-point Dixon VIBE sequence has additionally been found valuable for anatomic localization of PET-positive lesions, as the sequence is acquired over the whole field of view (i.e., all PET bed positions) and is nearly isotropic (19). Other approaches have been suggested for MRI-based AC, such as using a 3-dimensional T1-weighted sequence for a sequential PET/MRI scanner (20), an ultrashort echo time to identify the skull for brain imaging (21), and atlas-based techniques for potential identification of the bones (22).

After acquisition of the data for AC, the MRI component can be used to acquire any further sequences within the current field of view, simultaneously with the corresponding PET acquisition, which runs in parallel (Fig. 1). The MRI sequences can also extend beyond the PET acquisition time planned for the current bed position, with the rest of the examination being consequently delayed.

\section{Potential PET/MRI Protocols for Whole-Body Oncologic Staging}

For oncologic PET/MRI, one can envision 2 main scenarios that have important implications for designing the imaging protocol.

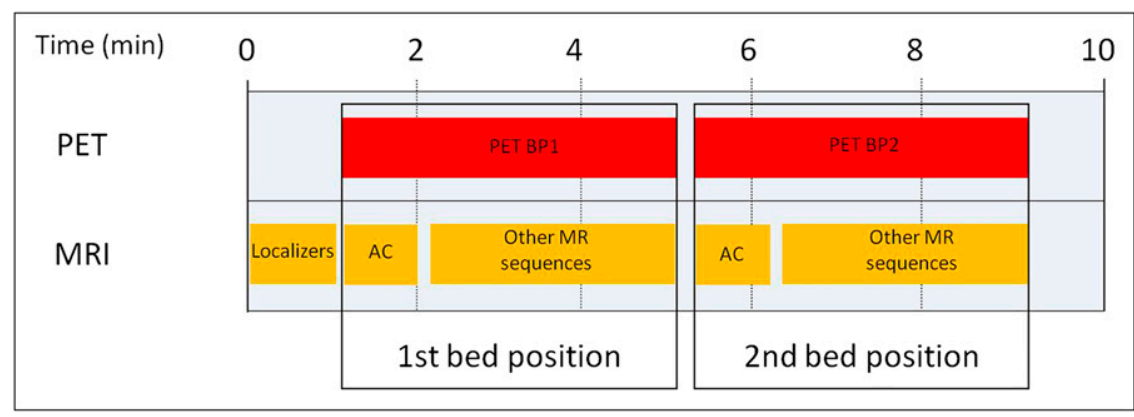

FIGURE 1. Diagram of basic acquisition protocol covering 2 bed positions in combined PET/MRI. For each PET bed position (4-min acquisition time in example), MRI AC sequence is acquired first and then MRI component can be used for further acquisitions without moving patient bed. BP1 = bed position 1; BP2 = bed position 2. 
On the one hand, patients may present with prior imaging results, such as a CT scan, and a partial- or whole-body PET/MRI scan of a specific region of interest may be required in addition to the existing imaging data. This scenario might be the case in a colorectal cancer patient in whom a solitary liver metastasis is found on staging CT. In this patient, a resection of the liver metastasis might be indicated, and to complete the staging, MRI of the liver would be requested to rule out further liver lesions and low-dose PET/CT to rule out distant metastases. With PET/ MRI, these questions could be answered in a 1-stop-shop examination. In this scenario, the focus of MRI would be only the liver, and a partial-body PET examination with 4-5 bed positions at 2-3 $\mathrm{min} /$ bed position could be performed quite quickly. During this time, only the Dixon AC sequence would be acquired (perhaps also an axial fast T2weighted half-Fourier single-shot turbo spin echo [HASTE] fat-saturated sequence), as previous staging with $\mathrm{CT}$ would already be available (Fig. 2). This protocol could be performed within $30 \mathrm{~min}$. The technical parameters of all discussed MRI sequences are presented in detail in Table 2.

On the other hand, many patients will present without extensive previous imaging data-for example, during follow-up for evaluation of an increase in tumor markers, to look for metastases or local recurrence. In this case, complete diagnostic coverage of the partial or whole body and specific areas of interest is required. Here, we describe 4 potential protocols for full diagnostic staging in oncology. The protocols cover either the head to the toes (whole body; e.g., for melanoma staging) or the base of the skull to the mid thigh (partial body), comparable to PET/CT, along with specific regions of interest depending on the patient's diagnosis (e.g., prostate cancer or head-and-neck cancer). Of course, many more protocols or individual variations are feasible, but these protocols encompass most adult oncologic examinations currently performed on the system.

For partial-body examinations, we start with imaging the trunk from the base of the skull to the thigh. Four PET bed positions are usually required, with an acquisition time of about $4 \mathrm{~min}$. This is slightly longer than for the first scenario but gives enough time-after acquisition of the initial breath-hold T1-weighted Dixon VIBE for AC- to obtain both breath-hold coronal T1-weighted TSE and fast axial T2-weighted HASTE fat-saturated dark-blood sequences. Depending on the patient's compliance and size, this part usually takes about 20-25 min. By this means, T1and T2-weighted contrast-enhanced sequences are obtained along with 2 planes, allowing both diagnosis of bone metastases and denomination of conditions such as simple cysts or enlarged lymph nodes. Reconstructions of the inphase image of the Dixon AC sequence in the sagittal and axial planes are done and analyzed in addition to the coronal T1-weighted TSE sequence, with the sagittal view being especially helpful for assessment of bone lesions in the spine.

This initial part of the partial-body examination is followed by imaging of a specific region of interest determined by the clinical question. We obtain a list-mode 15 - to 20-min PET scan with simultaneous acquisition of a dedicated MRI scan. For example, patients with head-and-neck cancer or thyroid cancer would undergo dedicated contrastenhanced MRI of the neck, providing information relevant for local and lymph node staging or for assessment of tumor recurrence, respectively (Figs. 3 and 4) (23). In restaging of prostate cancer with ${ }^{11} \mathrm{C}$-choline PET, the 15 - to 20 -min PET acquisition of the pelvis is accompanied by dedicated MRI sequences of this region (Fig. 5). To be more specific, a T2-weighted axial sequence is acquired to provide excellent anatomic detail. Diffusion-weighted imaging and dynamic contrast-enhanced MRI are performed mostly for evaluation of small local recurrences. Recent data imply that dynamic contrast-enhanced MRI is especially valuable for this purpose, as areas of local recurrence usually show early and intense contrast enhancement whereas in later phases scar tissue might also enhance, thus making detection of small tumors in the prostate bed difficult (Fig. 6).

Finally, each protocol includes an axial fat-saturated T1weighted gradient echo sequence of the whole lung, the abdomen, and the pelvis (e.g., T1-weighted VIBE), each during a single breath hold. Only 5 additional minutes of scanning time are needed, enabling, for example, the evaluation of lung metastases or the better delineation of enlarged lymph nodes, with a CT-like image impression.

FIGURE 2. Diagram of acquisition protocol for combined PET/MRI focusing on single area of interest (in this example, liver imaging), with only limited MRI evaluation of partial or whole body. ax = axial; $\mathrm{BP}=$ bed position; cor = coronal; $\mathrm{CM}=$ contrast medium; DWI = diffusion-weighted imaging; Dyn. = dynamic; fs = fat-saturated.

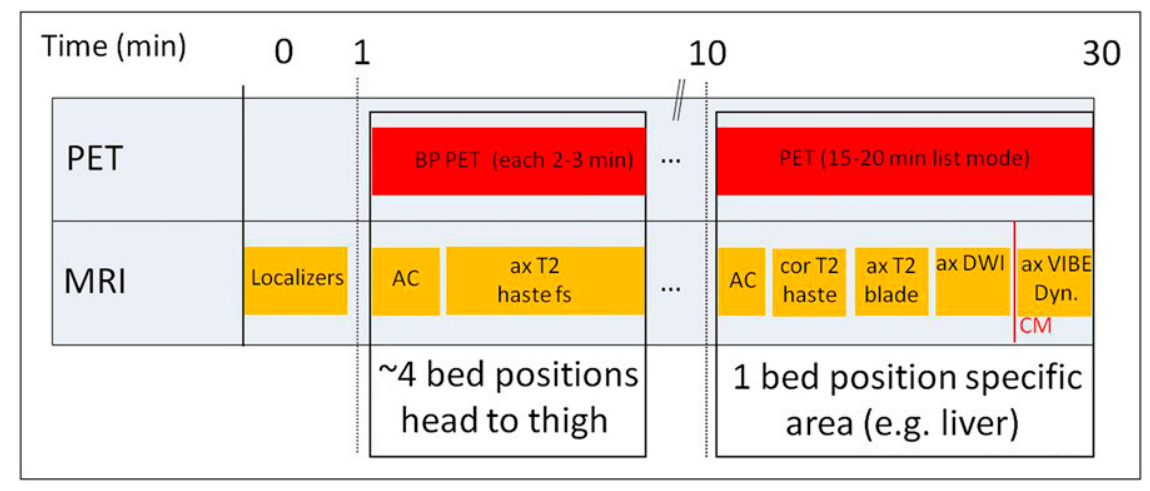




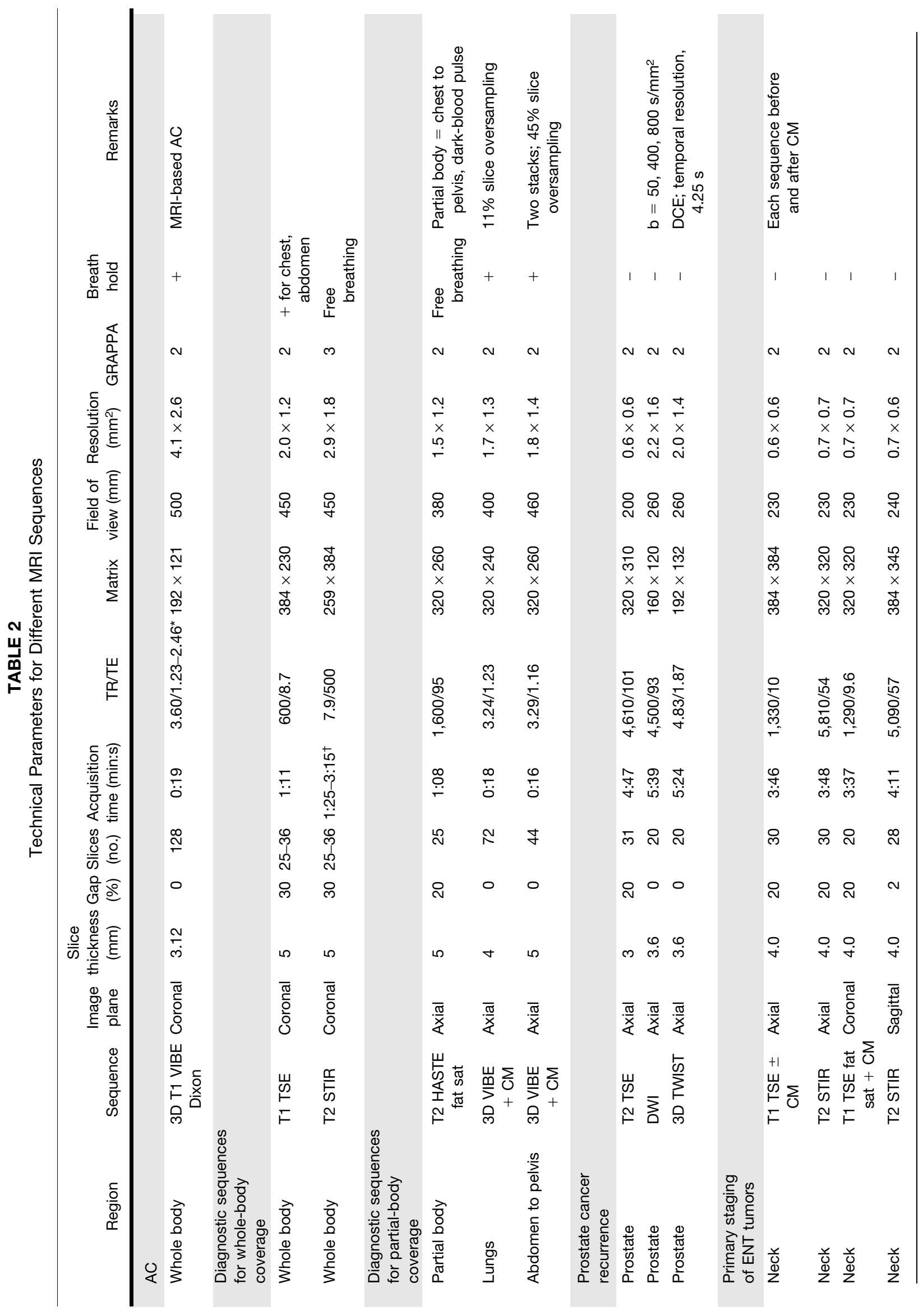




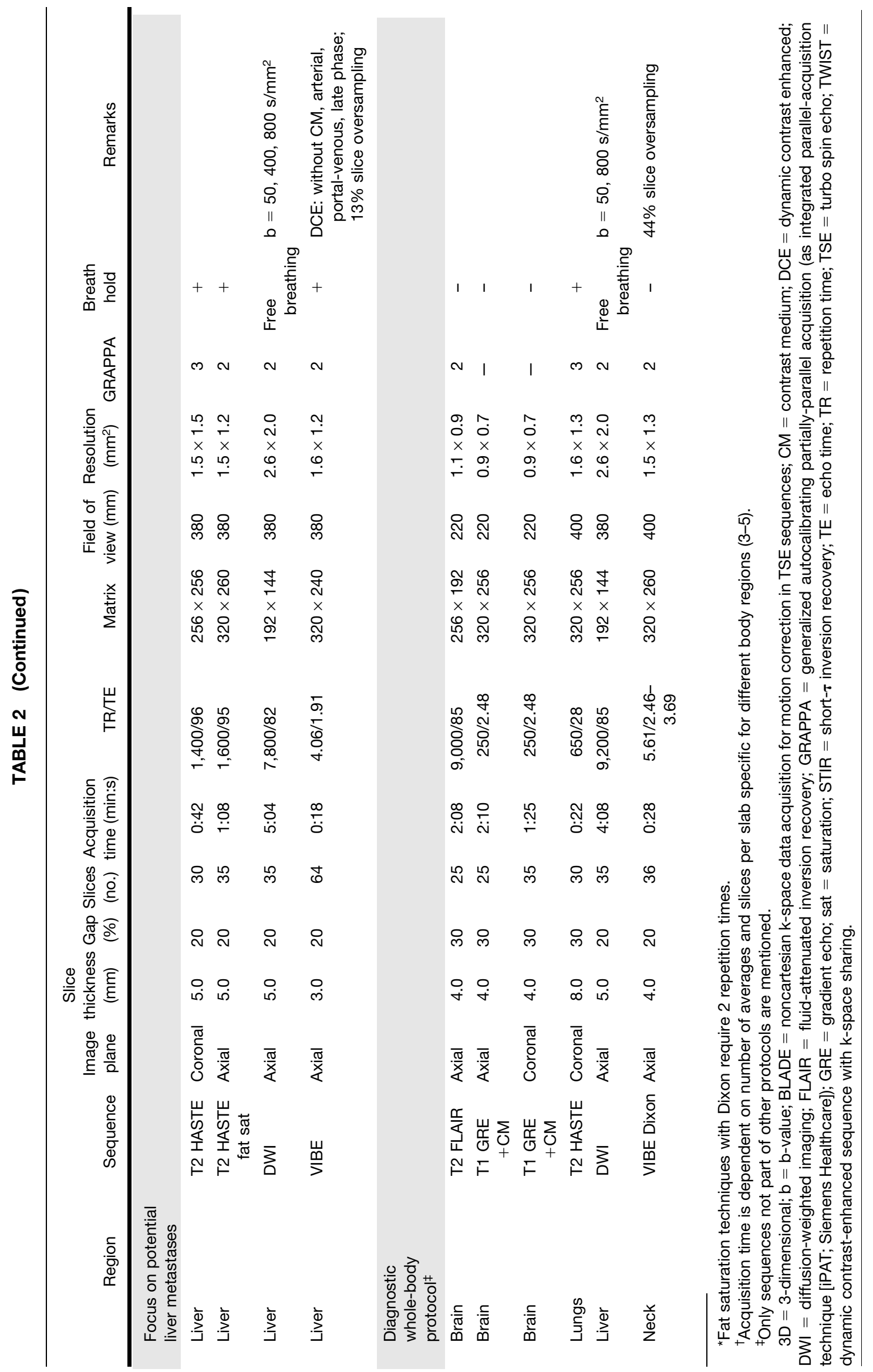

1420 The Journal of Nuclear Medicine • Vol. 53 • No. 9 • September 2012 


\begin{tabular}{|c|c|c|c|c|c|c|c|c|c|c|c|c|c|}
\hline \multicolumn{2}{|c|}{ Time $(\mathrm{min}) 0$} & \multicolumn{3}{|l|}{1} & \multicolumn{4}{|c|}{20} & \multicolumn{4}{|c|}{40} & 45 \\
\hline PET & & \multicolumn{3}{|c|}{ BP PET (each $4 \mathrm{~min}$ ) } & $\cdots$ & \multicolumn{5}{|c|}{ PET neck (20 min list mode) } & \multicolumn{3}{|c|}{ No PET } \\
\hline MRI & Localizers & $\mathrm{AC}$ & cor T1 TSE & $\begin{array}{c}\text { axT2 } \\
\text { hastefs }\end{array}$ & $\cdots$ & $A C$ & $\begin{array}{c}\text { ax T2 } \\
\text { stir }\end{array}$ & $\mathrm{axT1}$ & $\mathrm{ax} \mathrm{T1}$ & $\begin{array}{c}\text { cor T1 } \\
\text { fs }\end{array}$ & $\begin{array}{c}\text { ax } \\
\text { VIBE }\end{array}$ & $\begin{array}{c}\text { ax } \\
\text { VIBE }\end{array}$ & $\begin{array}{c}\text { ax } \\
\text { VIBE }\end{array}$ \\
\hline & & \multicolumn{3}{|c|}{$\begin{array}{l}\text { 3-4 bed positions } \\
\text { head to thigh }\end{array}$} & & & \multicolumn{4}{|c|}{$\begin{array}{l}1 \text { bed position } \\
\text { neck }\end{array}$} & \multicolumn{3}{|c|}{$\begin{array}{l}\text { Lungs to } \\
\text { pelvis }\end{array}$} \\
\hline
\end{tabular}

FIGURE 3. Diagram of acquisition protocol focusing on head-and-neck imaging in combined PET/MRI with full diagnostic coverage of partial or whole body. $a x=$ axial; $\mathrm{BP}=$ bed position; cor = coronal; $\mathrm{CM}=$ contrast medium; fs = fat-saturated.
No PET is acquired during this step. A potential limitation of the described protocols for head-and-neck or prostate cancer is that the liver is imaged only in the venous equilibrium phase, thus making detection of small liver lesions difficult. However, for patients with prostate cancer or thyroid cancer, liver metastases are rare and, when occurring, are usually within the context of diffuse metastatic disease and not single small lesions. In head-and-neck cancer patients, liver metastases can occur despite being relatively rare. The lack of portal-venous contrast-enhanced MRI information about the liver may be compensated for by the available ${ }^{18}$ F-FDG PET data, which have been demonstrated to be highly sensitive for assessment of metastatic disease, including liver lesions in patients with head-andneck cancer (24).

For patients with the focus on potential liver metastases, such as from breast cancer, colorectal cancer, or neuroendocrine tumors, we perform dedicated MRI of the liver, including dynamic contrast-enhanced sequences and diffusionweighted imaging (Figs. 7 and 8). Especially, diffusion-weighted imaging has evolved over the last few years to be a sensitive tool for detection of liver lesions and is superior to ${ }^{18} \mathrm{~F}-\mathrm{FDG}$ PET for lesions smaller than $1 \mathrm{~cm} \mathrm{(25).} \mathrm{Dynamic} \mathrm{contrast-}$ enhanced MRI is also a sensitive method and is useful for lesion classification as well (26). When indicated, central nervous system coverage is performed with axial fluidattenuated inversion recovery and contrast-enhanced T1weighted sequences. In addition, in this patient group axial fat-saturated T1-weighted gradient echo sequences are acquired, each during a single breath-hold (e.g., T1-weighted VIBE), as described above.

For certain indications, true whole-body coverage from head to toe or assessment of the central nervous system is mandatory, as for example, in melanoma patients or lung cancer patients. In these cases, we use a protocol with about 4 bed positions of 4 min each covering the torso and 3-5 additional bed positions of $2 \mathrm{~min}$ each covering the legs, depending on the size of the patient. During this time, coronal short- $\tau$ inversion recovery sequences (or T1weighted SE sequences) can be acquired simultaneously during the PET acquisition. For melanoma or tumors known to metastasize in unusual patterns, we prefer short- $\tau$ inversion recovery in this protocol as it better highlights softtissue lesions such as small lymph nodes or cutaneous lesions. Subsequently, MRI is performed only for liver, lung, and central nervous system coverage (Figs. 9 and 10). This protocol is, however, more time-consuming and requires about $60 \mathrm{~min}$ of scanning time (depending on the

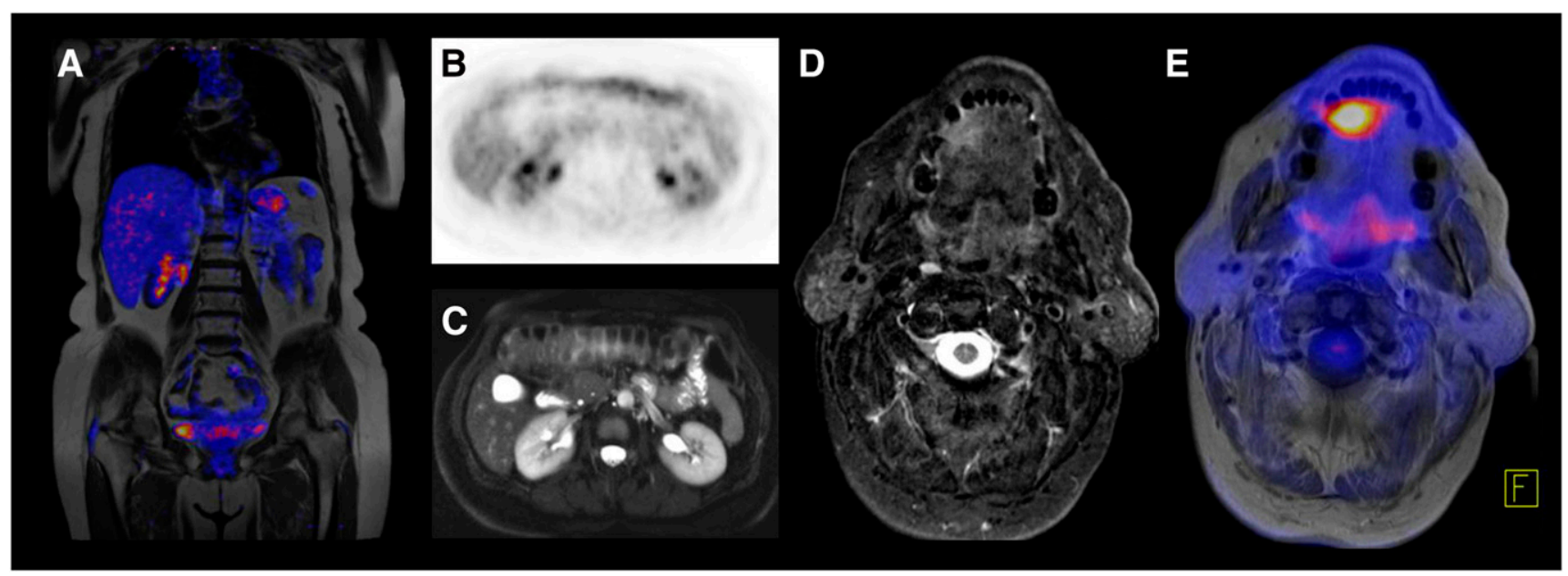

FIGURE 4. Example of head-and-neck imaging in combined PET/MRI ( ${ }^{18} \mathrm{~F}-\mathrm{FDG}$ ): patient with cancer of oral cavity. (A-C) Coronal T1-weighted turbo spin echo, axial PET, and axial T2-weighted fat-saturated HASTE images show no distant metastases. (D and E) T2-weighted hyperintense lesion on MRI with high glucose metabolism on right side in frontal oral cavity is suggestive of tumor. 
FIGURE 5. Diagram of acquisition protocol focusing on prostate imaging in combined PET/MRI with full diagnostic coverage of partial or whole body. $a x=a x-$ ial; $\mathrm{BP}=$ bed position; cor $=$ coronal; $\mathrm{CM}=$ contrast medium; fs $=$ fat-saturated.

\begin{tabular}{|c|c|c|c|c|c|c|c|c|c|c|c|c|}
\hline \multirow{3}{*}{$\begin{array}{l}\text { Time (min) } \\
\begin{array}{|c}\text { PET } \\
\text { MRI }\end{array}\end{array}$} & \multirow{3}{*}{\begin{tabular}{|l}
0 \\
\\
Localizers
\end{tabular}} & \multicolumn{3}{|c|}{1} & \multicolumn{4}{|c|}{20} & \multicolumn{3}{|c|}{35} & 4 \\
\hline & & \multicolumn{3}{|c|}{ BPPET (each $4 \mathrm{~min}$ ) } & ... & \multicolumn{4}{|c|}{ PET pelvis (15 min list mode) } & \multicolumn{3}{|c|}{ NOPET } \\
\hline & & $A C$ & cor T1 TSE & $\begin{array}{c}\text { ax T2 } \\
\text { hastefs }\end{array}$ & $\cdots$ & $A C$ & $\mathrm{ax} \mathrm{T2}$ & ax DWI & ax DCE & \begin{tabular}{|c} 
ax \\
VIBE
\end{tabular} & $\begin{array}{c}\text { ax } \\
\text { VIBE }\end{array}$ & $\begin{array}{c}\text { ax } \\
\text { VIBE }\end{array}$ \\
\hline & & \multicolumn{3}{|c|}{$\begin{array}{c}\sim 4 \text { bed positions } \\
\text { head to thigh }\end{array}$} & & & \multicolumn{3}{|c|}{$\begin{array}{c}1 \text { bed position } \\
\text { pelvis }\end{array}$} & \multicolumn{3}{|c|}{$\begin{array}{l}\text { Lungs to } \\
\text { pelvis }\end{array}$} \\
\hline
\end{tabular}

size and compliance of the patient), despite the advantage of having an integrated system.

In the system we used, the total-imaging-matrix surface coils do not cover the entire body in a normal-sized adult, and images of the lower legs are therefore not of optimal quality. If images of this area are of clinical relevance, the patient can be repositioned with feet first in the scanner to cover the lower legs with surface coils.

As a general diagnostic limitation of all the mentioned protocols, the inferiority of conventional MRI, in comparison with CT, for detecting small pulmonary nodules and assessing subtle parenchymal changes should be kept in mind. More specific MRI protocols in addition to the sequences described above, such as respiration-triggered or gated MRI sequences, can be included. However, these protocols may be time-consuming and probably can be efficiently applied only for specific lung-evaluation indications (currently under investigation). Alternatively, an unenhanced low-dose CT scan of the lungs might be added until systematic studies have addressed in more detail the performance of PET/MRI for evaluation of small lung metastases.

\section{Data Visualization and Analysis}

An unprecedentedly large number of images are generated in a hybrid PET/MRI examination, which-depending on the protocol—can generate more than 10,000 slices. As in the reading of PET/CT along with diagnostic CT, joint reading of PET/MRI by physicians from both specialties or by double-trained physicians is necessary, including those with subspecialties such as neuroradiology or pediatric radiology for certain indications. As for image analysis, there is no single strategy or algorithm for reading PET/MRI data, and every institution has to define its own way of doing so. However, some recommendations and suggestions for a potential reading workflow will be discussed here, with a focus on the special requirements of PET/ MRI data as compared with PET/CT data.

One basic difference between PET/MRI and PET/CT is that more than a single PET dataset might be available. For example, there may be a whole-body PET dataset acquired at 2-4 min per bed position, along with a separate PET bed position of 15-20 min covering a special region of interest (head and neck, prostate, liver, or another region). A further

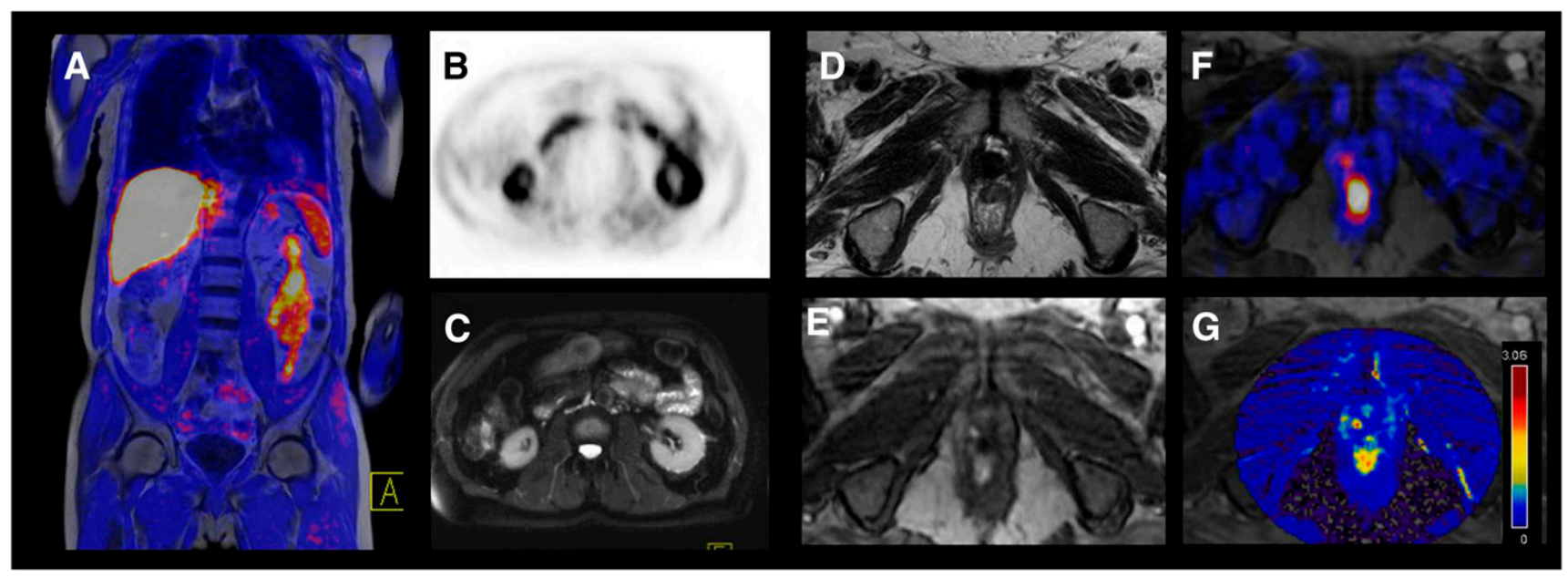

FIGURE 6. Example of prostate imaging in combined PET/MRI $\left({ }^{11} \mathrm{C}\right.$-choline): patient with radical prostatectomy and PSA recurrence. (A-C) Coronal T1-weighted turbo spin echo, axial PET, and axial T2-weighted fat-saturated HASTE images show no distant metastases. (D) Axial T2-weighted turbo spin echo image provides superb anatomic details of area of former prostate fossa. (E) Dynamic contrast-enhanced MR image shows early intense contrast enhancement in arterial phase. $(F)$ In fused ${ }^{11} \mathrm{C}$-choline PET image, moderate focal high uptake is found in corresponding region. (G) Parametric map indicating area under curve confirms early and intense enhancement of suspected lesion. 


\begin{tabular}{|c|c|c|c|c|c|c|c|c|c|c|c|}
\hline \multirow{2}{*}{$\begin{array}{l}\text { Time (min) } \\
\begin{array}{c}\text { PET }\end{array}\end{array}$} & \multirow[t]{2}{*}{0} & \multicolumn{3}{|l|}{1} & \multicolumn{2}{|c|}{20} & \multicolumn{4}{|c|}{40} & 45 \\
\hline & & \multicolumn{3}{|c|}{ BPPET (each 4 min) } & "W & \multicolumn{3}{|c|}{ PET liver (20 min list mode) } & \multicolumn{3}{|c|}{ No PET } \\
\hline \multirow[t]{2}{*}{ MRI } & Localizers & $\mathrm{AC}$ & cor T1 TSE & $\begin{array}{l}\text { ax T2 } \\
\text { hastefs }\end{array}$ & $\cdots$ & $A C$ & $\begin{array}{l}\text { cor T2 ax T2 ax DWI } \\
\text { haste hastefs }\end{array}$ & $\begin{array}{c}\text { ax VIBE } \\
\text { Dyn. }\end{array}$ & $\begin{array}{c}a x \\
\text { VIBE }\end{array}$ & $\begin{array}{c}a x \\
\text { VIBE }\end{array}$ & $\begin{array}{c}\text { ax } \\
\text { VIBE }\end{array}$ \\
\hline & & \multicolumn{3}{|c|}{$\begin{array}{c}\sim 4 \text { bed positions } \\
\text { head to thigh }\end{array}$} & & \multicolumn{3}{|c|}{$\begin{array}{c}1 \text { bed position } \\
\text { liver }\end{array}$} & \multicolumn{3}{|c|}{$\begin{array}{l}\text { Lungs to } \\
\text { pelvis }\end{array}$} \\
\hline
\end{tabular}

FIGURE 7. Diagram of acquisition protocol focusing on liver imaging in combined PET/MRI with full diagnostic coverage of partial or whole body. ax $=$ axial; $\mathrm{BP}=$ bed position; cor = coronal; $\mathrm{CM}=$ contrast medium; DWI = diffusion-weighted imaging; Dyn. = dynamic; fs = fat-saturated. difference is that there exist a multitude of different anatomic datasets, not all of which would necessarily cover the whole field of view or be isotropic, as opposed to modern multislice PET/CT data.

For interpretation of PET/MRI images, the PET data from the partial- or whole-body coverage are first fused with anatomic data, and a maximum-intensity projection of the PET data is created, just as in PET/CT. In a first navigation step, the Dixon $\mathrm{AC}$ sequence is used for image fusion: although its low resolution limits its diagnostic value, the advantage of near-isotropic coverage of the whole field of view is useful for a quick overview and for localization of PET-positive lesions. Subsequently, for a more detailed analysis, the T1-weighted coronal data and the HASTE T2-weighted fat-saturated axial data are then used for fusion with the whole-body PET images in the coronal and axial planes, respectively. These data are, however, not isotropic and thus are useful only in their primary orientation. They then can be compared side by side with the axial contrast-enhanced T1-weighted VIBE fat-saturated images for a complete analysis of the whole body. Afterward, the dedicated PET images of the area of interest, acquired for a longer time, can be fused with an anatomic dataset of this area and analyzed side by side with the other MRI sequences of the area.

It is even more important than in PET/CT that the nonattenuation-corrected images be evaluated during clinical reading of the PET images to recognize attenuation-related artifacts and to evaluate the lungs. In contrast to PET/CT, in PET/MRI it is mandatory that the MRI-based attenuation map (or $\mu$-map) be checked for any attenuation-related artifacts. The most common of these in PET/MRI are described in the following section.

\section{Possible Artifacts in PET/MRI}

The MRI component of a PET/MRI scanner should perform similarly to a standalone MRI scanner; thus, no particular artifacts are expected for the MRI part of the examination. However, the PET images can show artifacts resulting mainly from the use of MRI-based AC. Therefore, the artifacts in PET/MRI could differ from those in PET/CT.

Metals. Metallic implants produce a local signal loss in MRI images, misleading the image segmentation procedure and resulting in classification of the region as air instead of tissue in the MRI-based attenuation map. This misclassification may result in severely underestimated uptake in the

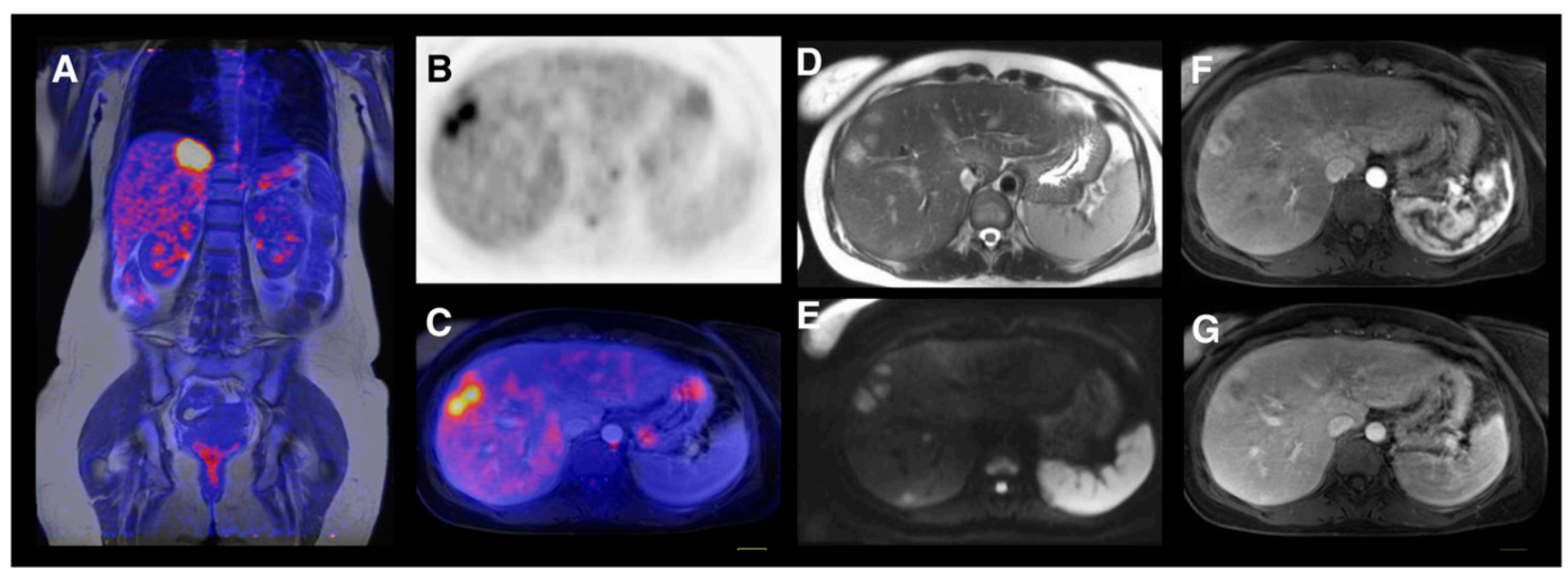

FIGURE 8. Example of liver imaging in combined PET/MRI ( $\left.{ }^{18} \mathrm{~F}-\mathrm{FDG}\right)$ : patient with breast cancer and liver metastases. (A) Fused coronal T1-weighted turbo spin echo image with PET image shows hypermetabolic lesion in liver below medial part of diaphragm. (B and C) Axial PET image and axial fused image outline 2 adjacent liver metastases in right lobe. (D-G) Axial morphologic MR images (T2-weighted HASTE $[D]$, diffusion-weighted [E], dynamic contrast-enhanced [F and G]) demonstrate high soft-tissue contrast on MRI and superb anatomic delineation of liver metastases. 


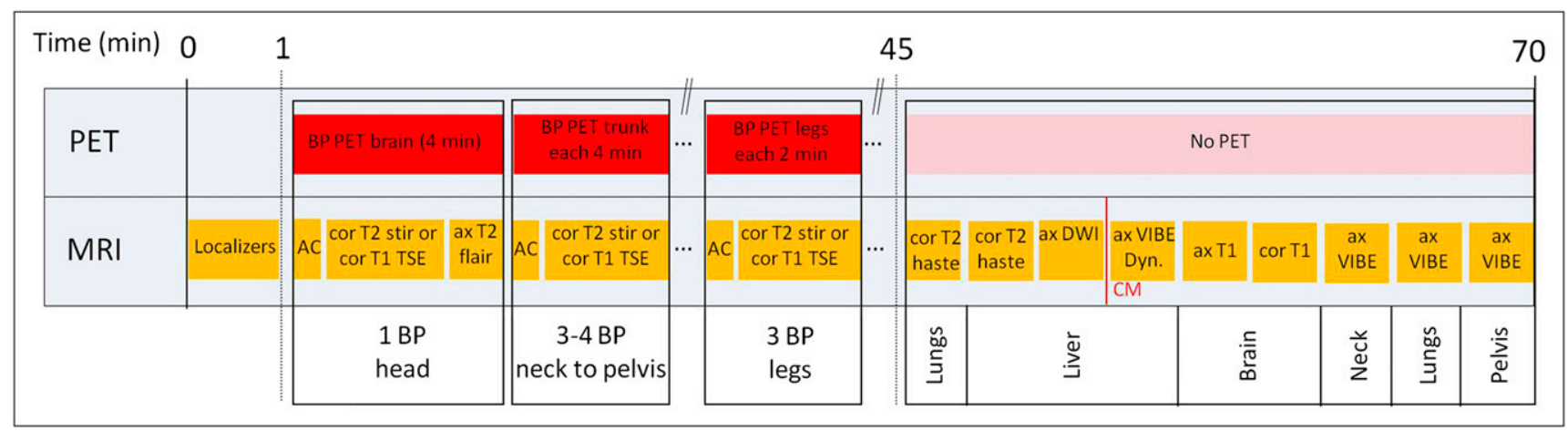

FIGURE 9. Diagram of fully diagnostic whole-body acquisition protocol in combined PET/MRI. ax = axial; BP = bed position; cor = coronal; $\mathrm{CM}=$ contrast medium; DWI = diffusion-weighted imaging; Dyn. = dynamic; $\mathrm{fs}=$ fat-saturated; stir = short- $\tau$ inversion recovery.

region surrounding the metallic implant (Fig. 11). The artifact differs from that observed in PET/CT, where metal usually leads to artificially increased uptake.

Truncation. Whereas the PET field of view is almost $60 \mathrm{~cm}$, the MRI field of view is only around $45 \mathrm{~cm}$ because the inhomogeneity of the magnetic field rolls off toward the edges of the bore. This limited field of view results in truncated parts of the body in MRI images in general and in the MRI-derived attenuation map in particular. If the patient is examined arms-up, truncation artifacts may occasionally be seen in the shoulders, breasts, abdomen, or hips. If the patient is examined arms-down, the arms of nearly all patients will be severely truncated in the images. A truncated attenuation map affects the PET image both through the AC and through the scatter correction (27,28). In our experience so far, the most noticeable effect is an anterior-posterior gradient in the liver for ${ }^{18} \mathrm{~F}$-FDG images (Fig. 12). Also, the quantification of the standardized uptake value of a lesion might be affected. For other tracers with stronger physiologic uptake in organs such as the liver
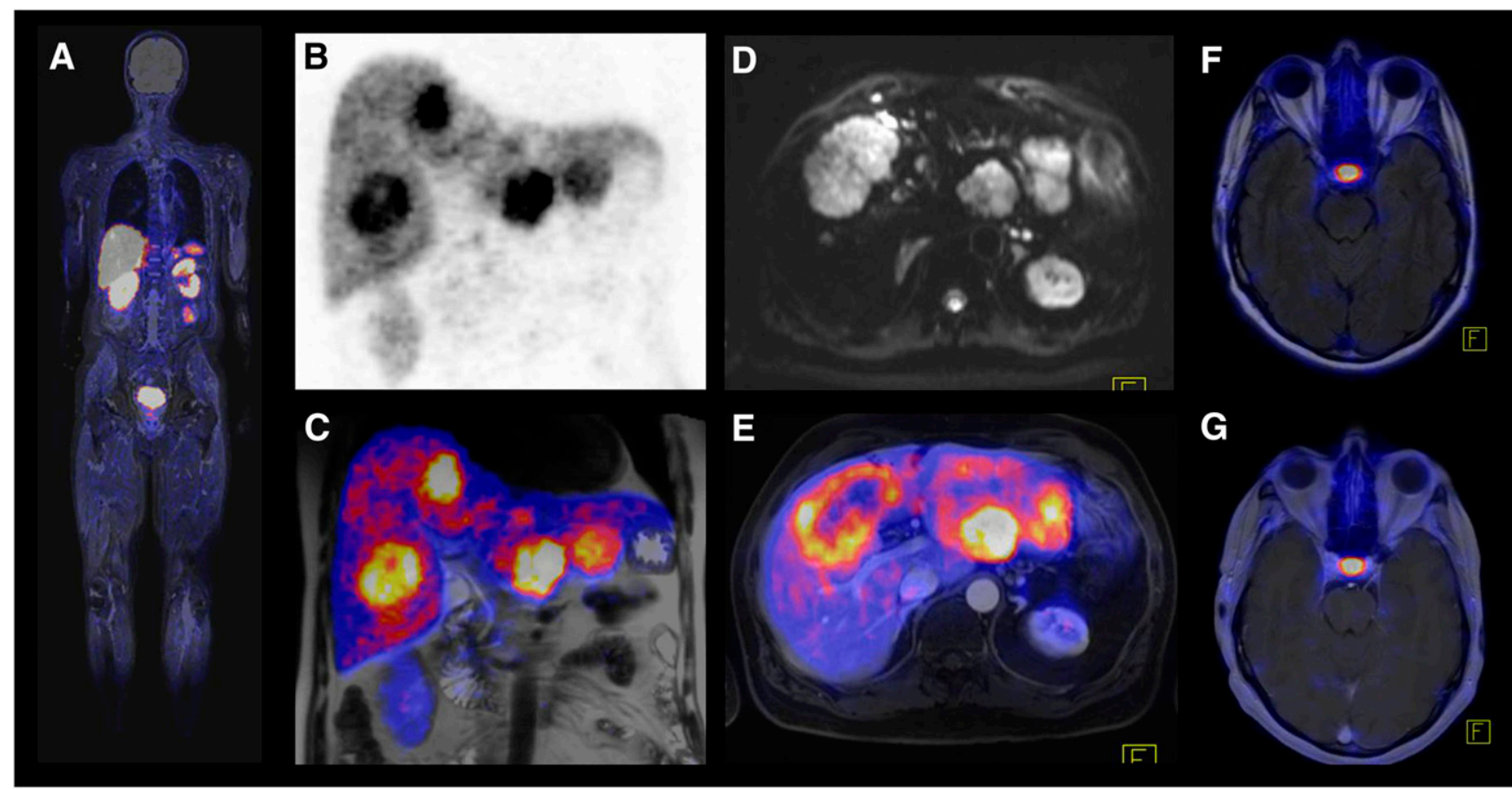

$\mathbf{G}$

F
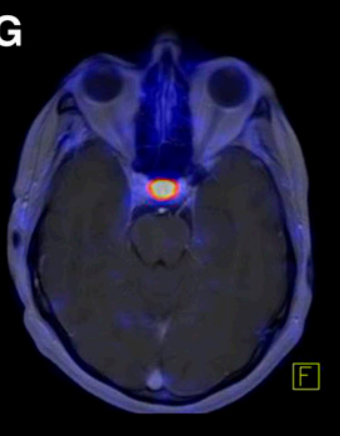

FIGURE 10. Example of whole-body imaging in combined PET/MRI ( $\left.{ }^{68} \mathrm{Ga}-\mathrm{DOTATOC}\right)$ : patient with neuroendocrine cancer and liver metastases. (A) Fused coronal T2-weighted short- $\tau$ inversion recovery and PET images demonstrate whole extent of examination. Because of incomplete coverage of lower legs by total-imaging-matrix surface coils, intensity and quality of MRI images in this area are substantially reduced. (B and $C$ ) PET and fused images show multiple liver metastases with high somatostatin receptor expression. (D) Diffusionweighted image (with b-value of $50 \mathrm{~s} / \mathrm{mm}^{2}$ ) shows large metastasis with many surrounding small lesions. (E) In comparison to diffusionweighted image, fused axial T2-weighted fat-saturated HASTE and PET image demonstrates that small lesions are not visible on PET. (F and G) Fused axial T2-weighted fluid-attenuated inversion recovery image and axial T1-weighted gadolinium-enhanced image show physiologic uptake of hypophysis and no evidence of brain metastases. 

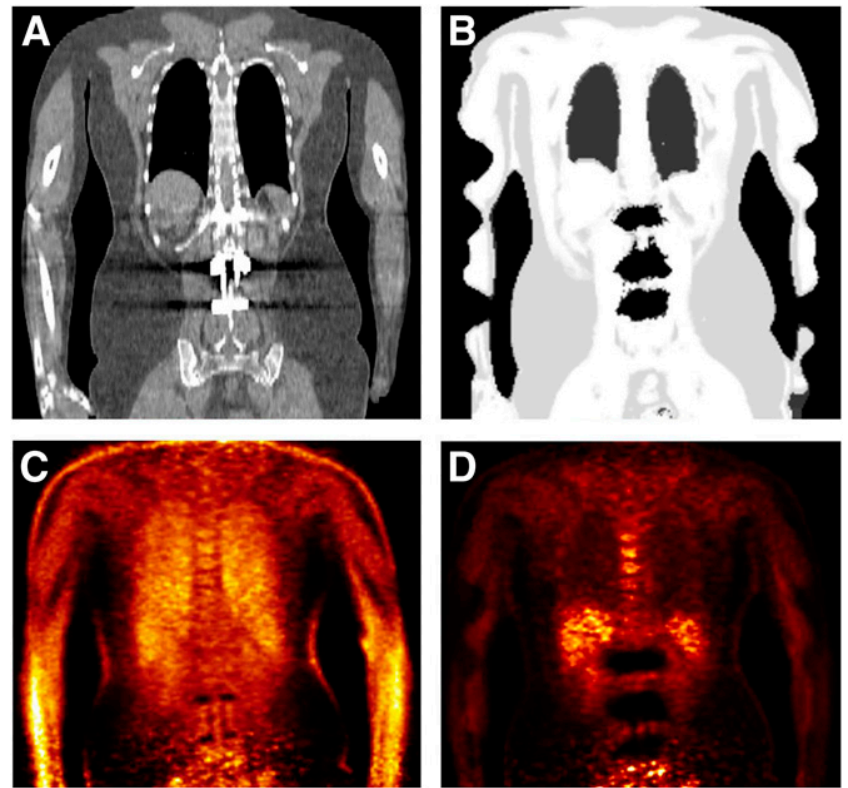

FIGURE 11. Patient with metallic implants from lumbar spine surgery. Metallic artifacts in CT image (A) result in region's being incorrectly segmented as air in MRI-based attenuation map (B). Non-AC PET image $(C)$ shows normal tracer distribution around implants, but MRI-based AC leads to large areas of underestimated uptake (D).

or spleen (e.g., ${ }^{11} \mathrm{C}$-choline or ${ }^{68} \mathrm{Ga}$-DOTATOC), the resulting artifact may occasionally be much harsher because of its impact on scatter correction, occasionally rendering the entire region near the respective organ unevaluable (Fig. 13), an effect that disappears for reconstructions without scatter correction, but at the price of losing the means of quantification. Techniques to recover the truncated part of the field of view have been developed $(29,30)$, and in the system we used it is possible to apply the PET emission data to roughly estimate the cropped part of the attenuation map. Such techniques might be helpful to minimize these artifacts, but further investigation and validation are still needed.

PET/MRI Misregistration. Misregistration between the PET emission data and the attenuation map is known to cause artifacts in the PET attenuation-corrected images $(31,32)$. In PET/MRI, simultaneous acquisition helps reduce the frequency of misregistration as compared with PET/CT, but misregistration artifacts are still occasionally present, mainly because of differences in the respiratory state. The attenuation sequence should be acquired in end expiration to achieve better alignment (31).

Lowered Uptake in Bone. Current methods for $\mathrm{AC}$ in PET/MRI ignore the specific contribution to attenuation by cortical bone. Consequently, decreased uptake is expected when MRI-based AC in used for images of massive bony structures (e.g., pelvis, spine, or femur) and their vicinity. Different groups evaluating MRI-based AC have reported maximum errors of up to $13 \%(18,20)$ or $17 \%$ (33) in the standardized uptake value of bony

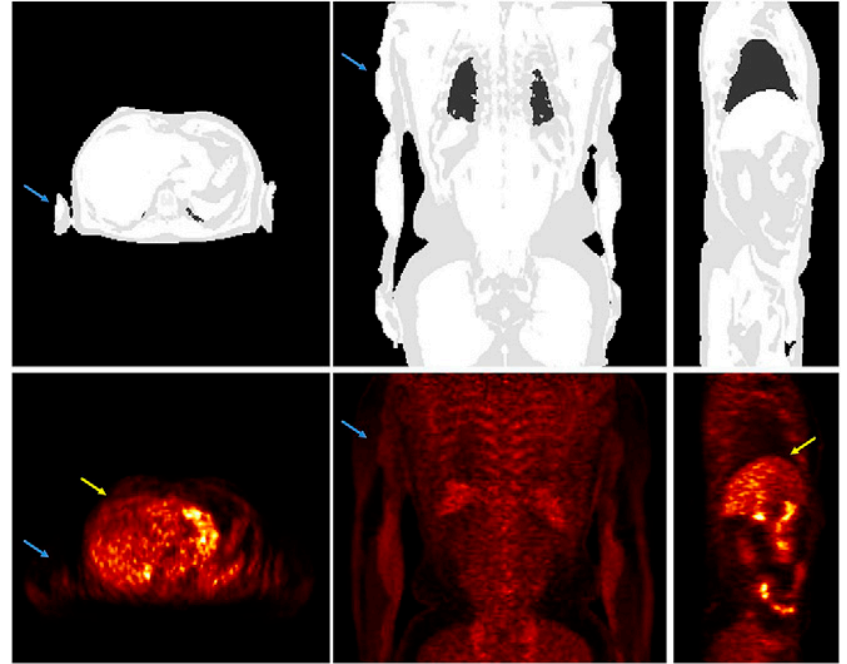

FIGURE 12. MRI-based attenuation map (top row) and corresponding ${ }^{18} \mathrm{~F}-\mathrm{FDG}$ PET images (bottom row) acquired arms-down showing truncation in arms and resulting artifacts: inhomogeneity in liver (anterior-posterior gradient, yellow arrows) and sharp change in uptake in arms corresponding to limit of field of view (blue arrows).

structures, but such errors should not greatly affect the diagnostic value of the technique.

Nonrecognition of Lung Compartment. The 2-point Dixon approach currently recognizes the lungs by a closedcomponent algorithm that detects the largest air-filled cavities and assigns to them the attenuation value for lung tissue. However, one or both lungs are occasionally not identified correctly and instead are interpreted as air, thus leading to undercorrection of standardized uptake values in this area. The possibility of this artifact can be assessed by referring to the attenuation map and the non-AC images. This approach is also useful for identifying the effects of contrast agents, which tend to produce extended contours.
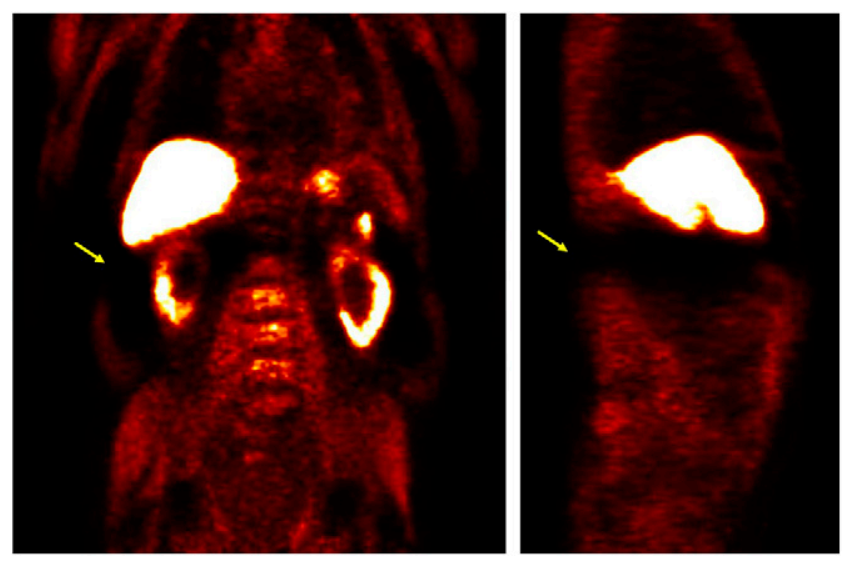

FIGURE 13. Severe artifacts in ${ }^{11} \mathrm{C}$-choline PET images acquired arms-down. Truncation of attenuation map can occasionally lead to biased scatter correction, which renders complete regions unevaluable (yellow arrows). 


\section{CONCLUSION}

Integrated PET/MRI systems allow simultaneous acquisition of PET and MRI data. Based on our initial experience over $1 \mathrm{y}$, the described considerations concerning workflow, imaging protocols, and data analysis show that the planning, execution, and analysis of whole-body PET/MRI in oncology can be quite time-consuming and complex. To guarantee a clinically valuable, time- and cost-efficient use of PET/MRI in oncology, it is mandatory that the indications be chosen correctly, that cross modality training be performed, that the acquisition protocols be optimized, and that the images be carefully reviewed, taking into account potential artifacts. However, if these points are respected, it is possible to perform high-quality whole-body PET/MRI in a reasonable time, giving the technique great potential for use in many indications in oncology.

\section{REFERENCES}

1. Buchanan M, Marsden PK, Mielke CH, Garlick PB. A system to obtain radiotracer uptake data simultaneously with NMR spectra in a high field magnet. IEEE Trans Nucl Sci. 1996;43:2044-2048.

2. Garlick PB, Marsden PK, Cave AC, et al. PET and NMR dual acquisition (PANDA): applications to isolated, perfused rat hearts. NMR Biomed. 1997; 10:138-142.

3. Shao Y, Cherry SR, Farahani K, et al. Simultaneous PET and MR imaging. Phys Med Biol. 1997;42:1965-1970.

4. Schlemmer HP, Pichler BJ, Schmand M, et al. Simultaneous MR/PET imaging of the human brain: feasibility study. Radiology. 2008;248:1028-1035.

5. Zaidi H, Ojha N, Morich M, et al. Design and performance evaluation of a whole-body Ingenuity TF PET-MRI system. Phys Med Biol. 2011;56:30913106.

6. Drzezga A, Souvatzoglou M, Eiber M, et al. First clinical experience of integrated whole-body PET/MR: comparison to PET/CT in patients with oncological diagnoses. J Nucl Med. 2012;53:845-855.

7. von Schulthess GK, Schlemmer HP. A look ahead: PET/MR versus PET/CT. Eur J Nucl Med Mol Imaging. 2009;36(suppl 1):S3-S9.

8. von Schulthess GK, Burger C. Integrating imaging modalities: what makes sense from a workflow perspective? Eur J Nucl Med Mol Imaging. 2010;37:980-990.

9. Nekolla SG, Martinez-Möller A, Saraste A. PET and MRI in cardiac imaging: from validation studies to integrated applications. Eur J Nucl Med Mol Imaging. 2009;36(suppl):S121-S130.

10. Heiss WD. The potential of PET/MR for brain imaging. Eur J Nucl Med Mol Imaging. 2009;36(suppl):S105-S112.

11. Hamblen SM, Lowe VJ. Clinical ${ }^{18} \mathrm{~F}-\mathrm{FDG}$ oncology patient preparation techniques. J Nucl Med Technol. 2003;31:3-7.

12. Schreve PD. Whole-body PET imaging methods. In: Valk PE, Bailey DL, Townsend DW, Maisey MN, eds. Positron Emission Tomography: Basic Science and Clinical Practice. 1st ed. New York, NY: Springer, 2004;481-493.

13. Barrington SF, Maisey MN. Skeletal muscle uptake of fluorine-18-FDG: effect of oral diazepam. J Nucl Med. 1996;37:1127-1129.
14. Mantlik F, Hofmann M, Werner MK, et al. The effect of patient positioning aids on PET quantification in PET/MR imaging. Eur J Nucl Med Mol Imaging. 2011;38:920-929.

15. Braun H, Ziegler S, Paulus DH, Quick HH. Hybrid PET/MRI imaging with continuous table motion. Med Phys. 2012;39:2735-2745.

16. Zaidi H. Is MR-guided attenuation correction a viable option for dual-modality PET/MR imaging? Radiology. 2007;244:639-642.

17. Hofmann M, Pichler B, Schölkopf B, Beyer T. Towards quantitative PET/MRI: a review of MR-based attenuation correction techniques. Eur J Nucl Med Mol Imaging. 2009;36(suppl 1):S93-S104.

18. Martinez-Möller A, Souvatzoglou M, Delso G. Tissue classification as a potential approach for attenuation correction in whole-body PET/MRI: evaluation with PET/CT data. J Nucl Med. 2009;50:520-526.

19. Eiber M, Martinez-Möller A, Souvatzoglou M, et al. Value of a Dixon-based MR/PET attenuation correction sequence for the localization and evaluation of PET-positive lesions. Eur J Nucl Med Mol Imaging. 2011;38:1691-1701.

20. Schulz V, Torres-Espallardo I, Renisch S, et al. Automatic, three-segment, MRbased attenuation correction for whole-body PET/MR data. Eur J Nucl Med Mol Imaging. 2011;38:138-152.

21. Catana C, van der Kouwe A, Benner T, et al. Toward implementing an MRIbased PET attenuation-correction method for neurologic studies on the MR-PET brain prototype. J Nucl Med. 2010;51:1431-1438.

22. Hofmann M, Bezrukov I, Mantlik F, et al. MRI-based attenuation correction for whole-body PET/MRI: quantitative evaluation of segmentation- and atlas-based methods. J Nucl Med. 2011;52:1392-1399.

23. Eiber M, Souvatzoglou M, Pickhard A, et al. Simulation of a MR-PET protocol for staging of head-and-neck cancer including Dixon MR for attenuation correction. Eur J Radiol. November 10, 2011 [Epub ahead of print].

24. Haerle SK, Schmid DT, Ahmad N, Hany TF, Stoeckli SJ. The value of ${ }^{18}$ F-FDG PET/CT for the detection of distant metastases in high-risk patients with head and neck squamous cell carcinoma. Oral Oncol. 2011;47:653-659.

25. Taouli B. Diffusion-weighted MR imaging for liver lesion characterization: a critical look. Radiology. 2012;262:378-380.

26. Eiber M, Fingerle AA, Brügel M, et al. Detection and classification of focal liver lesions in patients with colorectal cancer: retrospective comparison of diffusion-weighted MR imaging and multi-slice CT. Eur J Radiol. 2012;81: 683-691.

27. Beyer T, Bockisch A, Kühl H, Martinez MJ. Whole-body ${ }^{18}$ F-FDG PET/CT in the presence of truncation artifacts. J Nucl Med. 2006;47:91-99.

28. Delso G, Martinez-Möller A, Bundschuh R, Nekolla S, Ziegler SI. The effect of limited MR field of view in MR/PET attenuation correction. Med Phys. 2010;37:2804-2812.

29. Nuyts J, Michel C, Fenchel M, Bal G, Watson C. Completion of a truncated attenuation image from the attenuated PET emission data. Nuclear Science Symposium Conference Record (NSS/MIC). Piscataway, NJ: IEEE; 2010:2123-2127.

30. Salomon A, Goedicke A, Schweizer B, Aach T, Schulz V. Simultaneous reconstruction of activity and attenuation for PET/MR. IEEE Trans Med Imaging. 2011;30:804-813.

31. Beyer T, Antoch G, Blodgett T, Freudenberg LF, Akhurst T, Mueller S. Dualmodality PET/CT imaging: the effect of respiratory motion on combined image quality in clinical oncology. Eur J Nucl Med Mol Imaging. 2003;30:588-596.

32. Martinez-Möller A, Souvatzoglou M, Navab N, Schwaiger M, Nekolla SG. Artifacts from misaligned CT in cardiac perfusion PET/CT studies: frequency, effects, and potential solutions. J Nucl Med. 2007;48:188-193.

33. Keereman V, Van Holen R, Mollet P, Candenberghe S. The effect of errors in segmented attenuation maps on PET quantification. Med Phys. 2011;38:6010 6019 . 